Pacific Journal of Mathematics

A NOTE ON HOMOTOPY COMPLEX SURFACES WITH 


\title{
A NOTE ON HOMOTOPY COMPLEX SURFACES WITH NEGATIVE TANGENT BUNDLES
}

\section{B. WONG}

\begin{abstract}
We propose some problems concerning a weak rigidity phenomenon on compact complex manifolds with negative tangent bundles. Some observations have been made in the two dimensional case as an easy consequence of classification theory, and Yau's theorem on the rigidity of $\mathbb{C} P_{2}$. We point out that among the class of complex surfaces of general type with $c_{1}^{2}-c_{2}>0$ the cotangent dimension is a homotopy invariant possibly except in the case of $S^{2} \times S^{2}$.
\end{abstract}

1. Results and problems. A compact complex manifold $M$ with a negative tangent bundle $T(M)$ in the sense of Grauert if $T(M)$ is a strongly pseudoconvex manifold with the zero section as its only exceptional variety. This is the same thing as to say its cotangent bundle $T^{*}(M)$ is ample. If $X$ is a compact Kähler manifold with negative bisectional curvature, then $T(X)$ is negative by the formula of bicurvature [6]. This class of manifolds in general admit nontrivial local moduli $[13,14]$ unlike those Kähler manifolds with Nakanonegative curvatures which are locally rigid in the classical sense of Kodaira-Spencer [4, 10]. The aim of this paper is to propose a problem on a weak rigidity phenomenon of complex structures on compact complex manifolds with negative tangent bundles and to point out some observations in the complex two dimensional case.

Problem. Let $M$ be a compact Kähler manifold with negative bisectional curvature. Does it admit another complex structure, with the fixed topological (homotopic, or homeomorphic, or diffeomorphic) type, in which there exists a Kähler metric with positive definite Ricci tensor? A closely related problem is to ask whether the complex structures of negative tangent bundle and rational type are exclusive to each other.

An analogous open problem in differential topology and algebraic geometry is to ask whether general type and rational type complex structures can coexist on a compact four dimensional manifold with a fixed differentiable structure. In [1] R. Barlow proved that on a homeomorphic $\mathbb{C P}_{2}$ with eight points blown up, there exist both rational 
type and general type complex structures. Barlow's surface cannot carry a negative tangent bundle as a consequence of a simple computation of Chern numbers. On the other hand, a theorem of S. T. Yau determined that the homotopy $\mathbb{C} P_{2}$ admits only the standard complex structure [17]. Our problem is probably more natural from the angle of a curvature formulation of Schwarz's lemma [18] than the one stated above. We do not have any analytic or geometric technique to settle down our major problem at this point. Nevertheless, there is some hope that one can resolve the problem in the case of complex surfaces through an analysis of cotangent sheaf. We make several observations along this line based on a trick due to F. Bogomolov [3].

THEOREM 1. Let $X$ be a compact complex two-fold with a negative tangent bundle and $M$ be a minimal compact complex two-fold which is not diffeomorphic to the standard $S^{2} \times S^{2}$. Suppose $X$ and $M$ satisfy one of the following conditions:

(a) $X$ and $M$ have finite fundamental groups and they are homotopic to each other.

(b) $X$ and $M$ are homeomorphic.

Then the cotangent dimension of $M$ is equal to two.

The definition of cotangent dimension can be found in $\S 2$. It can be proved that a complex surface of cotangent dimension two must be of general type.

The following assertion follows from standard topological fact.

THEOREM 2. Let $X$ be a compact complex two-fold with a negative tangent bundle and $M$ be a minimal non-spin compact complex twofold. Suppose they satisfy one of the following two conditions:

(a) $X$ and $M$ are homotopic and their fundamental groups are finite.

(b) $X$ and $M$ are homeomorphic.

Then the cotangent dimension of $M$ is equal to two.

We recall here that a manifold has a spin structure iff its second Steifel-Whitney class $w_{2}=0$. To end our introduction, we would like to mention some open problems which should be clarified on our road.

Problem 1. Let $X$ be a compact complex two-fold with negative tangent bundle. Suppose $M$ is a compact complex two-fold homotopic (homeomorphic, diffeomorphic) to $X$. Is $T(M)$ negative? 
Problem 2. Does $S^{2} \times S^{2}$ admit a complex structure of general type (or even a complex structure with negative tangent bundle) upon the fixed underlying topological (diffeomorphic, homeomorphic, or homotopic) type?

Problem 3. Let $M$ be the compact four dimensional differentiable manifold obtained by $\mathbb{C} P_{2}$ blowing up one or two points (i.e., $\mathbb{C} P_{2} \# \overline{\mathbb{C} P_{2}}$ or $\mathbb{C} P_{2} \# 2 \overline{\mathbb{C} P_{2}}$, where $\overline{\mathbb{C} P_{2}}=\mathbb{C} P_{2}$ with opposite orientation, "\#" means connected sum). Does $M$ admit a complex structure of general type (or even a complex structure with negative tangent bundle)?

Problem 4. Let $X$ be a compact complex manifold covered by a bounded domain in $\mathbb{C}^{2}$. Is the cotangent dimension of $X$ equal to two? Suppose $M$ is a compact complex two-fold homotopic (or homeomorphic, or diffeomorphic) to $X$; is the cotangent dimension equal to two?

Problem 5. Is the property that the cotangent dimension is equal to two for complex surfaces a homotopic (homeomorphic, diffeomorphic) invariant?

Problem 6. Explore all our problems to higher dimensional situations.

2. Background materials and related results. Let $V$ be a compact complex manifold and $D$ be a divisor, the $D$-dimension according to Iitaka [11] is the number

$$
K(D, V)= \begin{cases}\max \operatorname{dim} \Phi_{m D}(V) & \text { if } N(D, V) \neq \phi, \\ -\infty & \text { if } N(D, V)=\phi,\end{cases}
$$

where $N(D, V)=\left\{m>0 \mid \operatorname{dim}_{\mathbb{C}} H^{0}(V, m D) \geq 1\right\}$ and $\Phi_{m D}$ is the canonical meromorphic mapping defined by a basis $\left\{\Phi_{0}, \Phi_{1}, \ldots\right.$, $\left.\Phi_{n}\right\}$ of $H^{0}(V, m D)$, namely

$$
\begin{aligned}
\Phi_{m D}: V & \rightarrow \mathbb{C} P^{n} \\
z & \rightarrow\left(\Phi_{0}(z): \Phi_{1}(z): \cdots: \Phi_{n}(z)\right)
\end{aligned}
$$

(in homogeneous coordinates). 
The canonical dimension (or Kodaira dimension) $K(V)$ of $V$ is defined to be $K(D, V)$ when $D$ is chosen as the canonical divisor $K_{V}$ of $V$. This is an invariant intrinsically attached to $V$. A compact complex manifold $V$ is of general type iff $K(V)=\operatorname{dim}_{\mathbb{C}} V$. Another equivalent characterization is that

$$
\varlimsup_{k \rightarrow \infty} \frac{P_{k}(V)}{k^{m}}>0
$$

where $m=\operatorname{dim}_{\mathbb{C}} V, P_{k}(V)=\operatorname{dim}_{\mathbb{C}} H^{0}\left(V, k K_{V}\right)$.

Let $E$ be a holomorphic vector bundle of rank $r$ over a compact complex manifold $X, \operatorname{dim}_{\mathbb{C}} X=m$. We denote by $S^{n}(E)$ the $n$th symmetric power of $E$. Let $P(E)$ be the projective bundle of the hyperplane through the origin in the fibers of $E$. We denote by $\pi: P(E) \rightarrow X$ the natural projection and $O_{P(E)}(1)$ the tautological line bundle on $P(E)$. There are canonical isomorphisms

$$
\pi_{*}: O_{P(E)}(n) \rightarrow S^{n}(E)
$$

and

$$
H^{0}\left(P(E), O_{P(E)}(n)\right) \cong H^{0}\left(X, S^{n} E\right), \quad \text { for } n \geq 0 .
$$

$E$ is ample iff $O_{P(E)}(1)$ is an ample line bundle over $P(E)$ in the usual sense of algebraic geometry. From the viewpoint of pseudoconvexity in several complex variables, it is equivalent to $E^{*}$ being (Grauert) negative (i.e., $E^{*}$ is a strongly pseudoconvex manifold with the zero section as its only exceptional variety). If $E$ is ample over $X$, then a standard argument in algebraic geometry and several complex variables implies that $H^{0}\left(X, S^{n} E\right)$ has enough sections to yield an embedding of $X$ into a Grassmannian, for sufficiently large $n$.

Let us now assume $E$ is a rank two negative holomorphic vector bundle over a complex surface $V$. Following from a result of Kleiman [7], the Chern numbers satisfy $C_{1}^{2}(E)>0, C_{2}(E)>0$ and $C_{1}^{2}(E)-$ $C_{2}(E)>0$, where $C_{1}(E)$ and $C_{2}(E)$ are the first and second Chern classes of $E$. In particular, if the tangent bundle of $V$ is negative, the Chern numbers of $V$ will satisfy $C_{1}^{2}>0, C_{2}>0$, and $C_{1}^{2}-C_{2}>0$; here $C_{1}$ and $C_{2}$ are the Chern classes of $V$. The following lemma, due to Bogomolov [3], will play an important role in our discussions. For the convenience of the readers and for the completion of this paper, we include the proof here.

LEMMA 2.1 ([3]). Let $E$ be an r-dimensional holomorphic vector bundle over a compact complex two-fold $V$ s.t. $C_{1}^{2}(E)-C_{2}(E)>0$. 
Then either $\operatorname{dim}_{\mathbb{C}} H^{0}\left(V, S^{n} E\right)$ or $\operatorname{dim}_{\mathbb{C}} H^{0}\left(V, S^{n} E^{*}\right)$ grows faster than $\mathrm{cn}^{r+1}$, for some constant $c>0$.

In particular, if $E$ is the tangent bundle $T(V)$, then either $\operatorname{dim}_{\mathbb{C}} H^{0}\left(V, S^{n} T\right)$ or $\operatorname{dim}_{\mathbb{C}} H^{0}\left(V, S^{n} T^{*}\right)$ grows faster than $\mathrm{cn}^{3}$.

Proof. By the Hirzebruch-Riemann-Roch theorem and a computation due to Bogomolov [3], one has the following identity:

$$
\begin{aligned}
\chi\left(V, S^{n} E\right)= & \operatorname{dim}_{\mathbb{C}} H^{0}\left(V, S^{n} E\right)-\operatorname{dim}_{\mathbb{C}} H^{1}\left(V, S^{n} E\right) \\
& +\operatorname{dim}_{\mathbb{C}} H^{2}\left(V, S^{n} E\right) \\
= & -p_{r+1}(n) C_{2}+\frac{r+2 n-1}{2(r+1)} p_{r}(n) C_{1}^{2} \\
& +p_{r-1}(n+1) \frac{K^{2}+\chi}{12}-\frac{1}{2} p_{r}(n) C_{1} K,
\end{aligned}
$$

where $p_{n_{1}}\left(n_{2}\right)=n_{2}\left(n_{2}+1\right) \cdots\left(n_{2}+n_{1}-1\right) / n_{1} !, K=$ canonical class of $V, \chi=$ Euler class of $V, C_{1}=$ first Chern class of $E$, and $C_{2}=$ second Chern class of $E$.

We observe that as $n$ tends to infinity, the terms $p_{r+1}(n)$ and $\frac{r+2 n-1}{2(r+1)} p_{r}(n)$ will dominate. Both of these two terms are polynomials in $n$ with degree $r+1$. As $n$ grows to infinity, we can write

$$
\begin{gathered}
\operatorname{dim}_{\mathbb{C}} H^{0}\left(V, S^{n} E\right)-\operatorname{dim}_{\mathbb{C}} H^{1}\left(V, S^{n} E\right)+\operatorname{dim}_{\mathbb{C}} H^{2}\left(V, S^{n} E\right) \\
=\frac{2 n+r-1}{2(r+1)} p_{r}(n) C_{1}^{2}-p_{r+1}(n) C_{2} \\
\quad+\{\text { terms of polynomials in } n \text { of degree }<r+1\} .
\end{gathered}
$$

We further observe that

$$
\lim _{n \rightarrow \infty} \frac{p_{r+1}(n)}{\frac{2 n+r-1}{2(r+1)} p_{r}(n)}=\lim _{n \rightarrow \infty} \frac{2(n+r)}{(2 n+r-1)}=1 .
$$

With all the above information we can write

$$
\chi\left(S^{n} E\right)=p_{r+1}(n)\left(C_{1}^{2}-C_{2}\right)+o\left(n^{r+1}\right),
$$

where $\lim _{r \rightarrow \infty}\left(o\left(n^{r+1}\right) / n^{r+1}\right)=0$. Thus we have the inequality

$$
\operatorname{dim}_{\mathbb{C}} H^{0}\left(V, S^{n} E\right)+\operatorname{dim}_{\mathbb{C}} H^{2}\left(V, S^{n} E\right)>a \cdot n^{r+1}+o\left(n^{r+1}\right),
$$

where $a=\left(C_{1}^{2}-C_{2}\right)(1-\varepsilon) /(r+1) !, C_{1}^{2}-C_{2}>0,0<\varepsilon<1$. By Serre duality $H^{2}\left(V, S^{n} E\right) \cong H^{0}\left(V, K \otimes S^{n} E\right)$, we have $\operatorname{dim}_{\mathbb{C}} H^{0}\left(V, S^{n} E\right)+\operatorname{dim}_{\mathbb{C}} H^{0}\left(V, K \otimes S^{n} E^{*}\right)>a \cdot n^{r+1}+o\left(n^{r+1}\right)$. 
We observe that the minimal model $\tilde{V}$ of $V$ must also satisfy the inequality $C_{1}^{2}-C_{2}>0$. By [2, Table 10 , pp. 188], we see that $\widetilde{V}$ has to be algebraic. Hence $V$ is also algebraic. We can therefore choose a divisor $D$ on $V$ so that both $D$ and $D-K$ are effective. This gives an exact sequence which arises from the restriction of $S^{n} E^{*} \otimes K$ to $D$,

$$
\left.0 \rightarrow S^{n} E^{*} \otimes K \otimes(-D) \rightarrow S^{n} E^{*} \otimes K \rightarrow S^{n} E^{*} \otimes K\right|_{D} \rightarrow 0 .
$$

This associates to an exact sequence of cohomologies,

$$
\begin{aligned}
0 & \rightarrow H^{0}\left(V, S^{n} E^{*} \otimes K \otimes(-D)\right) \rightarrow H^{0}\left(V, S^{n} E^{*} \otimes K\right) \\
& \rightarrow\left(D,\left.S^{n} E^{*} \otimes K\right|_{D}\right) \rightarrow H^{1}\left(V, S^{n} E^{*} \otimes K \otimes(-D)\right) \rightarrow \cdots .
\end{aligned}
$$

This implies that

$$
\begin{aligned}
\operatorname{dim}_{\mathbb{C}} H^{0}(V, & \left.S^{n} E^{*} \otimes K\right)-\operatorname{dim}_{\mathbb{C}} H^{0}\left(V, S^{n} E^{*} \otimes K \otimes(-D)\right) \\
& \leq \operatorname{dim}_{\mathbb{C}} H^{0}\left(D,\left.S^{n} E^{*} \otimes K\right|_{D}\right) .
\end{aligned}
$$

By the Riemann-Roch theorem over curves, one obtains

$$
\operatorname{dim}_{\mathbb{C}} H^{0}\left(D,\left.S^{n} E^{*} \otimes K\right|_{D}\right) \leq \alpha \cdot n^{r}+\beta,
$$

where $\alpha$ and $\beta$ are positive constants. Nevertheless, if $D-K$ is effective, one has the inequality

$$
\operatorname{dim}_{\mathbb{C}} H^{0}\left(V, S^{n} E^{*} \otimes K \otimes(-D)\right) \leq \operatorname{dim}_{\mathbb{C}} H^{0}\left(V, S^{n} E^{*}\right) .
$$

We observe that

$$
\operatorname{dim}_{\mathbb{C}} H^{0}\left(V, S^{n} E^{*} \otimes K\right)-\operatorname{dim}_{\mathbb{C}} H^{0}\left(V, S^{n} E^{*}\right) \leq \alpha \cdot n^{r}+\beta .
$$

Combining the arguments above for sufficiently large $n$ and for $c=$ $C_{1}^{2}-C_{2}(1-\varepsilon) /(r+1)$ !, one finally concludes with the following desired inequality

$$
\operatorname{dim}_{\mathbb{C}} H^{0}\left(V, S^{n} E\right)+\operatorname{dim}_{\mathbb{C}} H^{0}\left(V, S^{n} E^{*}\right)>c \cdot n^{r+1} .
$$

DEFINITION. Let $E$ be a rank $r$ holomorphic vector bundle over a compact complex manifold $X$. The $E$-dimension of $E$ over $X$ is the number

$$
e(E, X)= \begin{cases}d-r+1 & \text { if } d \neq-\infty \\ -r & \text { if } d=-\infty\end{cases}
$$

where $d=$ the $D$-dimension of $O_{P(E)}(1)$ over $P(E)$.

Definition. The cotangent dimension of $X$, namely $\operatorname{Cod}(X)$, is the number $e(E, X)$ when $E$ is the cotangent bundle of $X$. 
We state some remarks concerning cotangent dimension and refer to [9] for their proofs.

REMARK 2.1. It is immediate from the definition that $\operatorname{Cod}(X)$ takes one of the numbers from $\left\{-\operatorname{dim}_{\mathbb{C}} X, \ldots, 0, \ldots, \operatorname{dim}_{\mathbb{C}} X\right\}$.

REMARK 2.2 [9]. The cotangent dimension is a bimeromorphic invariant.

REMARK 2.3 [9]. It follows basically from the classification of complex surfaces that for an algebraic surface $X$, the inequality $\operatorname{Cod}(X) \leq$ $K(X)$ holds if $K(X) \geq 0$. In particular, for a compact complex twofold $M$, if $\operatorname{Cod}(M)=2$, then it is of general type. Here we should notice that for any compact complex two-fold $M$, if $\operatorname{Cod}(M)=2$, then it admits two independent meromorphic functions. Hence $M$ must be algebraic by an old theorem of Chow and Kodaira [5].

REMARK 2.4 [9]. Let $M_{1}$ and $M_{2}$ be two compact complex manifolds such that $M_{1}$ is an unramified holomorphic cover of $M_{2}$. Then $\operatorname{Cod}\left(M_{1}\right)=\operatorname{Cod}\left(M_{2}\right)$.

The following observation is an easy consequence of the classification theory of complex surfaces.

THEOREM 2.2. Let $M$ be a compact complex two-fold with $C_{1}^{2}-$ $C_{2}>0$. Then $M$ is either of general type with cotangent dimension two or of rational type.

For the proof we observe that one can read from [3, Table 10, pp. 188] that a minimal compact complex two-fold with $C_{1}^{2}-C_{2}>0$ can only be rational or of general type. On the other hand if $M$ satisfies $C_{1}^{2}-C_{2}>0$, its minimal model must also satisfy the same inequality. The cotangent dimension is equal to two is a consequence of Lemma 2.1.

\section{Proofs of Theorems 1 and 2.}

(A) Proof of Theorem 1. Case (a). Let $\widetilde{M}$ and $\tilde{X}$ be the universal coverings of $M$ and $X$ respectively. Since $\pi_{1}(M)$ and $\pi_{1}(X)$ are finite, $\widetilde{M}$ and $\widetilde{X}$ are compact complex manifolds. It follows from assumption that $\widetilde{M}$ and $\widetilde{X}$ must be homotopic to each other. It is a consequence of a theorem in [15], [8], [16] that the Hirzebruch index of $\widetilde{M}$ and $\widetilde{X}$ must be equal. The index of a compact complex twofold is equal to $\frac{1}{3}\left(C_{1}^{2}-2 C_{2}\right)$. Moreover, it is elementary to prove 
that the Euler number for an oriented simply connected four-fold is a homotopy invariant. We have therefore proved that $C_{1}^{2}$ and $C_{2}$ for $\widetilde{M}$ and $\widetilde{X}$ are identical. The tangent bundle $T(\widetilde{X})$ is negative because $X$ is assumed to be so. By a theorem of Kleiman [7], $C_{2}(\tilde{X})>0$ and $C_{1}^{2}(\widetilde{X})-C_{2}(\widetilde{X})>0$. It follows that $C_{1}(\widetilde{M})>0, C_{2}(\widetilde{M})>0$, and $C_{1}^{2}(\widetilde{M})-C_{2}(\widetilde{M})>0$. Moreover, one has immediately $C_{1}^{2}(M)>0$, $C_{2}(M)>0$ and $C_{1}^{2}(M)-C_{2}(M)>0$ because $\widetilde{M}$ is a finite unramified cover of $M$. From Lemma 2.1, Remark 2.3, and Theorem 2.2, $M$ is either of general type with cotangent dimension two or of rational type. (This also follows from the classification theory of complex surfaces, namely [2, Table 10, p. 188]. This is a fact that a minimal compact complex two-fold with $C_{1}^{2}>0$ and $C_{2}>0$ must be of general type or of rational type. But it should be noticed the "minimal assumption" has been used here if you apply this alternate argument.) Finally, a minimal rational two-fold is either diffeomorphic to $\mathbb{C} P_{2}$ or to $S^{2} \times S^{2}$. However, by Yau's theorem [17] $X$ cannot be homotopic to $\mathbb{C} P_{2}$ because $X$ has a negative tangent bundle. The possibility for being $S^{2} \times S^{2}$ is also dropped by the assumption in Theorem 1 .

Case (b). It is a known fact that the Chern numbers for compact complex two-folds are homeomorphic invariants (for a proof, we refer to [2, Theorem 2.6, p. 116]). The other part of the proof is then a line to line copy of part (a).

(B) Proof of Theorem 2. The proof is parallel to (A). The only new ingredient is the fact that $S^{2} \times S^{2}$ is a spin manifold.

\section{REFERENCES}

[1] R. Barlow, A simply connected surface of general type with $p_{g}=0$, Invent. Math., 79 (1985), 293-301.

[2] Barth-Peters-Van de Ven, Compact Complex Surfaces, Ergebnisse, Band 4, Springer-Verlag, Berlin, 1984.

[3] F. A. Bogomolov, Holomorphic tensors and vector bundles on projective varieties; Math. USSR Izvestija, 13 (1979), 499-555.

[4] E. Calabi-Vessentini, On compact locally symmetric Kähler manifolds, Ann. of Math., 71 (1960), 472-507.

[5] Chow-Kodaira, On analytic surfaces with two independent meromorphic functions, Proc. Nat. Acad. Sci. U.S.A., 38 (1952), 319-325. 
[6] Griffiths, Hermitian differential geometry, Chern class and positive vector bundles, in Global Analysis, Univ. of Tokyo Press and Princeton Univ. Press, (1969), 185-251.

[7] S. Kleiman, Ample vector bundles on algebraic surfaces, Proc. Amer. Math. Soc., 21 (1969), 673-676.

[8] J. Milnor, On simply-connected 4-manifolds, in Symp. Int. de Topology Alg., Univ. Mexico, Mexico, (1958), 122-128.

[9] F. Sakai, Symmetric powers of the cotangent bundle and classification of algebraic varieties, in Lecture Notes in Math. No. 732, Springer-Verlag, New York, 545563.

[10] Y.-T. Siu, The complex-analyticity of harmonic maps, vanishing and Lefschetz theorems, J. Differential Geom., 17 (1982), 55-138.

[11] K. Ueno, Classification of Algebraic Varieties and Compact Complex Spaces, Lecture Notes in Math. No. 439, Springer-Verlag, Heidelberg, 1975.

[12] B. Wong, A class of compact complex manifolds with negative tangent bundles, in Math. Z., 185 (1984), 217-223.

[13] _ The uniformization of compact Kähler surfaces with negative curvature, J. Differential Geom., 16 (1981), 407-420.

[14] __ A global moduli space of ample subvarieties on compact Kähler manifolds with very negative curvature, Math. Ann., 277 (1987), 605-615.

[15] J. H. C. Whitehead, On simply-connected 4-dimensional polyhedra, Comment. Math. Helv., 22 (1949), 48-92.

[16] C. T. C. Wall, On simply-connected 4-manifolds, J. London Math. Soc., 39 (1964), 141-149.

[17] S. T. Yau, Calabi's conjecture and some new results in algebraic geometry, Proc. Nat. Acad. Sci. U.S.A., 74 (1977), 1789-1799.

[18] _ A general Schwarz lemma for complete Kähler manifolds, Amer. J. Math., 100 (1978), 197-203.

Received July 7, 1990. Research partially supported by NSF Grant.

UNIVERSITY OF CALIFORNIA

RIVERSIDE, CA 92521 



\section{PACIFIC JOURNAL OF MATHEMATICS EDITORS}

\author{
V. S. VARADARAJAN \\ (Managing Editor) \\ University of California \\ Los Angeles, CA 90024-1555-05 \\ Herbert Clemens \\ University of Utah \\ Salt Lake City, UT 84112 \\ THOMAS ENRIGHT \\ University of California, San Diego \\ La Jolla, CA 92093
}

Nicholas ERcolani

University of Arizona

Tucson, AZ 85721

R. FINN

Stanford University

Stanford, CA 94305

VAUGHAN F. R. JONES

University of California

Berkeley, CA 94720

STEVEN KeRCKHOFF

Stanford University

Stanford, CA 94305

\section{C. MOORE \\ University of California \\ Berkeley, CA 94720}

MARTIN SChaRLEMANN

University of California

Santa Barbara, CA 93106

HAROLd STARK

University of California, San Diego

La Jolla, CA 92093

\section{ASSOCIATE EDITORS}

\begin{tabular}{|c|c|c|c|c|}
\hline R. ARENS & $\begin{array}{l}\text { E. F. BECKENBACH } \\
(1906-1982)\end{array}$ & NeumanN & $\begin{array}{l}\text { F. WoLF } \\
(1904-1989)\end{array}$ & K. YoshidA \\
\hline \multicolumn{5}{|c|}{ SUPPORTING INSTITUTIONS } \\
\hline \multirow{2}{*}{\multicolumn{2}{|c|}{$\begin{array}{l}\text { IVERSITY OF ARIZONA } \\
\text { IVERSITY OF BRITISH COLUMBIA }\end{array}$}} & UNIVERS & Y OF OREGON & \\
\hline & & UNIVERS & OF SOUTHER & RNIA \\
\hline \multicolumn{2}{|c|}{ IFORNIA INSTITUTE OF TECHNOLOGY } & STANFOI & UNIVERSITY & \\
\hline \multirow{2}{*}{\multicolumn{2}{|c|}{$\begin{array}{l}\text { IVERSITY OF CALIFORNIA } \\
\text { NTANA STATE UNIVERSITY }\end{array}$}} & UNIVERS & OF HAWAII & \\
\hline \multirow{2}{*}{\multicolumn{2}{|c|}{$\begin{array}{l}\text { NTANA STATE UNIVERSITY } \\
\text { IVERSITY OF NEVADA, RENO }\end{array}$}} & UNIVERS & Y OF TOKYO & \\
\hline & & UNIVERS & OF UTAH & \\
\hline \multirow{2}{*}{\multicolumn{2}{|c|}{$\begin{array}{l}\text { N MEXICO STATE UNIVERSITY } \\
\text { EGON STATE UNIVERSITY }\end{array}$}} & WASHING & N STATE UNI & RSITY \\
\hline & & UNIVERS & OF WASHING & \\
\hline
\end{tabular}

The Supporting Institutions listed above contribute to the cost of publication of this Journal, but they are not owners or publishers and have no responsibility for its content or policies.

Mathematical papers intended for publication in the Pacific Journal of Mathematics should be in typed form or offset-reproduced (not dittoed), double spaced with large margins. Please do not use built up fractions in the text of the manuscript. However, you may use them in the displayed equations. Underline Greek letters in red, German in green, and script in blue. The first paragraph must be capable of being used separately as a synopsis of the entire paper. In particular it should contain no bibliographic references. Please propose a heading for the odd numbered pages of less than 35 characters. Manuscripts, in triplicate, may be sent to any one of the editors. Please classify according to the 1991 Mathematics Subject Classification scheme which can be found in the December index volumes of Mathematical Reviews. Supply name and address of author to whom proofs should be sent. All other communications should be addressed to the managing editor, or Elaine Barth, University of California, Los Angeles, California 90024-1555-05.

There are page-charges associated with articles appearing in the Pacific Journal of Mathematics. These charges are expected to be paid by the author's University, Government Agency or Company. If the author or authors do not have access to such Institutional support these charges are waived. Single authors will receive 50 free reprints; joint authors will receive a total of 100 free reprints. Additional copies may be obtained at cost in multiples of 50 .

The Pacific Journal of Mathematics (ISSN 0030-8730) is published monthly except for July and August. Regular subscription rate: $\$ 190.00$ a year (10 issues). Special rate: $\$ 95.00$ a year to individual members of supporting institutions.

Subscriptions, orders for numbers issued in the last three calendar years, and changes of address should be sent to Pacific Journal of Mathematics, P.O. Box 969, Carmel Valley, CA 93924, U.S.A. Old back numbers obtainable from Kraus Periodicals Co., Route 100, Millwood, NY 10546.

The Pacific Journal of Mathematics at P.O. Box 969, Carmel Valley, CA 93924 (ISSN 0030-8730) is published monthly except for July and August. Second-class postage paid at Carmel Valley, Californiä 93924, and additional mailing offices. Postmaster: send address changes to Pacific Journal of Mathematics, P.O. Box 969, Carmel Valley, CA 93924.

\section{PUBLISHED BY PACIFIC JOURNAL OF MATHEMATICS, A NON-PROFIT CORPORATION}




\section{Pacific Journal of Mathematics}

\section{Vol. 151, No. $2 \quad$ December, 1991}

Michael G. Eastwood and A. M. Pilato, On the density of twistor

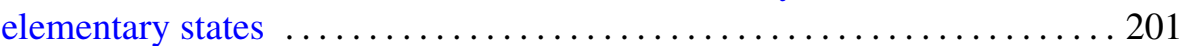

Brian E. Forrest, Arens regularity and discrete groups $\ldots \ldots \ldots \ldots \ldots \ldots 217$

Yu Li Fu, On Lipschitz stability for F.D.E ..................... 229

Douglas Austin Hensley, The largest digit in the continued fraction expansion of a rational number $\ldots \ldots \ldots \ldots \ldots \ldots \ldots \ldots \ldots \ldots \ldots . \ldots 237$

Uwe Kaiser, Link homotopy in $\mathbb{R}^{3}$ and $S^{3}$

Ronald Leslie Lipsman, The Penney-Fujiwara Plancherel formula for abelian symmetric spaces and completely solvable homogeneous

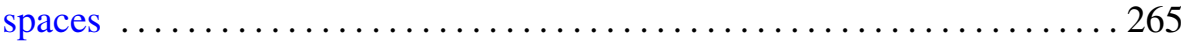

Florin G. Radulescu, Singularity of the radial subalgebra of $\mathscr{L}\left(F_{N}\right)$ and the

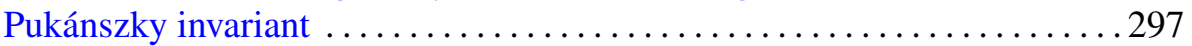

Albert Jeu-Liang Sheu, The structure of twisted SU(3) groups $\ldots . \ldots \ldots . \ldots 307$

Morwen Thistlethwaite, On the algebraic part of an alternating link . . . . . 317

Thomas (Toma) V. Tonev, Multi-tuple hulls .................. 335

Arno van den Essen, A note on Meisters and Olech's proof of the global asymptotic stability Jacobian conjecture ..................... 351

Hendrik J. van Maldeghem, A characterization of the finite Moufang hexagons by generalized homologies

Bun Wong, A note on homotopy complex surfaces with negative tangent bundles

Chung-Tao Yang, Any Blaschke manifold of the homotopy type of $\mathbf{C} P^{n}$ has the right volume 